\title{
Obturator Hernia - an Uncommon Cause of Small Bowel Obstruction
}

\author{
Kamal Galketiya • Srikanth Sakrepatna • Sivakumar Gananadha
}

Received: 12 September 2012 / Accepted: 12 October 2012 /Published online: 7 November 2012

(C) 2012 The Society for Surgery of the Alimentary Tract

Keywords Hernia $\cdot$ Obturator hernia $\cdot$ Small bowel obstruction

\section{Case Report}

A 79-year-old female was admitted to the emergency department. She gave a 72-h history of vomiting, colicky abdominal pain, abdominal distension, and constipation. Systems review revealed weight loss of $5 \mathrm{~kg}$ over 12 months but was otherwise unremarkable. A colonoscopy 3 months earlier had found no significant abnormalities and the past surgical history included an open appendicectomy, bilateral tubal ligation, and umbilical hernia repair.

On clinical examination, the vital signs were within normal limits and the abdomen was distended and non-tender. There were no obvious hernias to be found at clinical examination. An abdominal X-ray was suggestive of distal small bowel obstruction. A CT scan of the abdomen and pelvis was performed.

The CT scan showed multiple dilated fluid-filled small bowel loops measuring up to $40 \mathrm{~mm}$ in maximal diameter. A transition point was present in the distal ileum at the level of a right obturator hernia containing

K. Galketiya $(\bowtie) \cdot$ S. Sakrepatna $\cdot$ S. Gananadha Department of Surgery, The Canberra Hospital, Australian National University Medical School,

Canberra, ACT 2605, Australia

e-mail:kamaldanielle@gmail.com a distal ileal small bowel loop (Fig. 1a, b). The small bowel distal to the hernia was collapsed and decompressed. There was no evidence of intraperitoneal free fluid or pneumoperitoneum.

At laparotomy, a strangulated right obturator hernia was present. The hernia was reduced. The small bowel was necrotic and perforated on reduction. A small bowel resection and anastomosis was performed. The hernia defect was obliterated using 2-0 prolene monofilament suture. Mesh was not used due to the bowel perforation. Postoperatively, the nasogastric tube was removed on the second day and oral intake was commenced. Bowel function returned on the fourth postoperative day. The patient was discharged on the eight postoperative day to a respite facility due to her age and general frailty. She was reviewed at 4 weeks in the community with no postoperative complications present.

\section{Discussion}

Obturator herniae are rare. ${ }^{1}$ Incidence rates of obturator herniae vary widely throughout the world with Asian studies showing higher rates in comparison to Western studies. $^{2}$

It is a significant cause of small intestinal obstruction especially in emaciated elderly women. ${ }^{3}$ The clinical signs are often nonspecific and the diagnosis can be difficult. $^{2}$ A palpable groin mass is not common in an obturator hernia because the hernia mass is usually concealed beneath the pectineus. ${ }^{3}$ An obturator hernia produces signs due to compression of the obturator nerve and includes the Howship-Romberg sign and the Hannington-Kiff sign. These may be overlooked, 

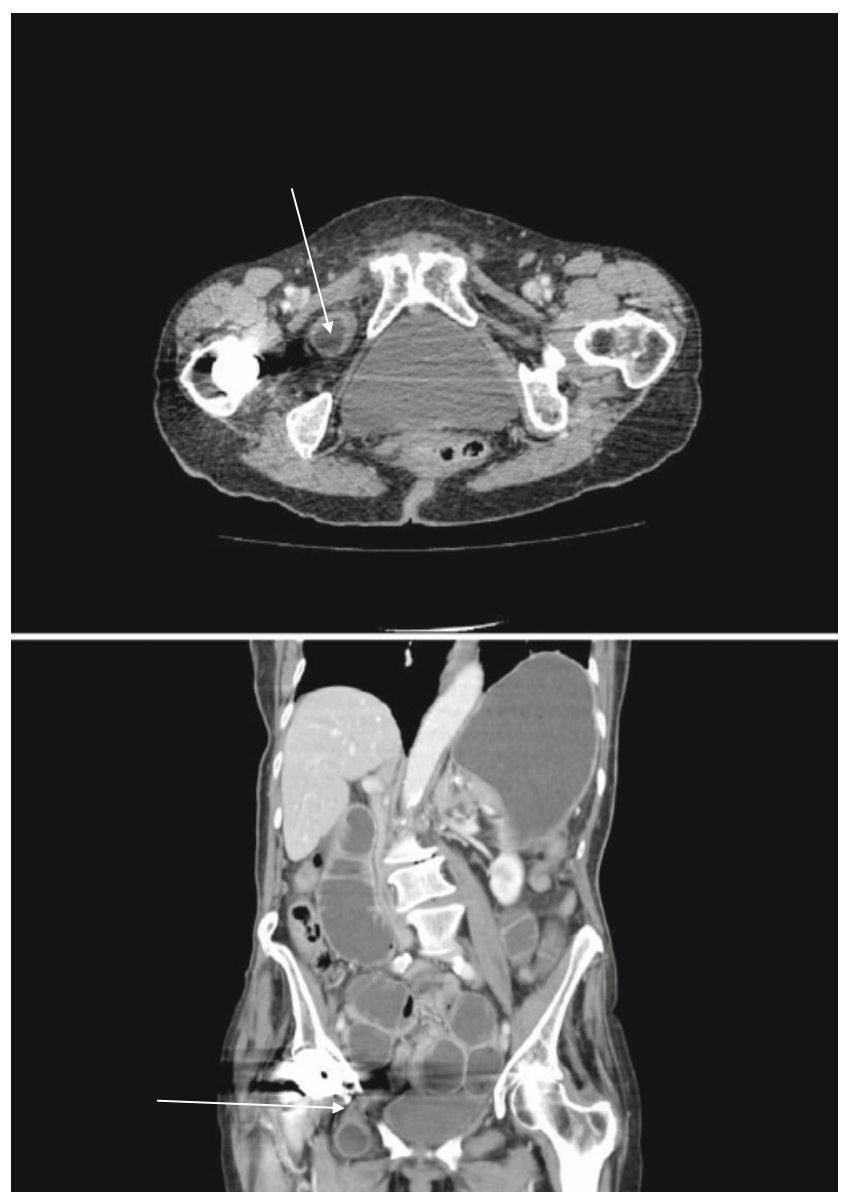

Fig. 1 a Axial CT scan showing the obturator hernia on the right side (arrow). The hernia contains a fluid-filled loop of small bowel between pectineus and obturator externus muscles. b Coronal CT scan showing the obturator hernia on the right side (arrow). There is a fluid-filled loop of small bowel herniating through the obturator foramen misinterpreted, or masked by the abdominal signs and symptoms. $^{3}$ The Howship-Romberg sign is pain radiating along the distribution of the obturator nerve along the medial aspect of the thigh to the knee, exacerbated by extension, adduction, or medial rotation of the hip, and relieved by hip flexion. ${ }^{3}$ The Hannington-Kiff sign is an absent thigh adductor reflex in association with an obturator hernia. ${ }^{4}$

There is often a delay in diagnosis and surgical intervention with an associated high morbidity and mortality noted in obturator hernia. ${ }^{3}$ Computed tomographic imaging provides an excellent means of preoperative diagnosis. ${ }^{1}$ Operative repair of obturator herniae may be performed via a preperitoneal approach, intraabdominal approach (laparotomy), laparoscopic transabdominal approach, or a laparoscopic extraperitoneal approach., ${ }^{2,3}$ Interrupted sutures or synthetic mesh can be used to obliterate the hernia defect. ${ }^{2}$

\section{References}

1. Nasir BS, Zendejas B, Ali SM, Groenewald CB, Heller SF, Farley DR. Obturator hernia: the Mayo Clinic experience. Hernia 2012; 16:315-319

2. Mantoo SK, Mak K, Tan TJ. Obturator hernia: diagnosis and treatment in the modern era. Singapore Med J 2009; 50:866870

3. Shen-Shin C, Yan-Shen S, Yih-Jyh L, Yun-Sheng T, Pin-Wen L. A Review of Obturator Hernia and a Proposed Algorithm for its Diagnosis and Treatment. World J. Surg. 2005; 29:450-454

4. Hannington-Kiff JG. Absent thigh adductor reflex in obturator hernia. Lancet 1980; 1:180 